\title{
INNOVATION AND IMPROVEMENT \\ Clinic First: Prioritizing Primary Care Outpatient Training for Family Medicine Residents at Group Health Cooperative
}

\author{
Kathleen Barnes, MD, MPH $H^{7}$ and Carl G Morris, MD, MPH ${ }^{1,2}$ \\ 'Group Health Cooperative Family Medicine Residency, Group Health Cooperative, Seattle, WA, USA; ${ }^{2}$ Department of Family Medicine, University \\ of Washington, Seattle, WA, USA.
}

KEY WORDS: primary care; ambulatory care; curriculum/program evaluation.

J Gen Intern Med 30(10): 1557-60

DOI: $10.1007 / \mathrm{s} 11606-015-3272-\mathrm{Z}$

(c) Society of General Internal Medicine 2015

\begin{abstract}
I met Beth-Ann, a sixty-one-year-old female, during my first clinic as an intern. She was newly insured and looking for treatment for persistent heart palpitations and bright red blood in her stool. Her medical chart was peppered with poorly controlled chronic diseases, including diabetes, hypertension, and fibromyalgia. "I' $m$ in need of a tune-up," she said. Sucking in my breath, I tried to hide how overwhelmed I felt as our visit began.
\end{abstract}

Ask any primary care resident how they chose their specialty and chances are that longitudinal relationships with patients are their central motivation. In surprising contrast, primary care residencies follow an inpatient-centric model of training, with residents spending less than $20 \%$ of training in the outpatient setting where these relationships are fostered. ${ }^{1}$ Consequently, residents lack both the number and frequency of hours in clinic to develop the skills to provide continuous care for a panel of patients. ${ }^{1}$

Moreover, the meager time spent in outpatient training is frequently of poor quality. Insufficient funding combined with the infrequent and irregular clinic presence of residents severely cripples the provision of well-coordinated outpatient care. This training model deprives residents of the personal fulfillment that comes from providing high quality longitudinal primary care, and it deflates expectations of what is achievable in the clinic setting. ${ }^{1}$

As a second-year Family Medicine resident at Group Health Cooperative (GHC) in Seattle, I am part of an experiment in resident education that seeks to change the prevailing model of primary care training. Our hypothesis is that to best equip and engage future primary care physicians, the first priority in training must be to provide continuous care for a panel of patients in a highly functional outpatient setting. To pursue this goal, we completely redesigned our curriculum. We refer to

Published online July 30, 2015 the transformed curriculum as our 'Clinic First' model of family medicine residency training.

Before discussing the curriculum changes, it is important to recognize that our residency clinic lies within an integrated delivery system centered on an advanced primary care model. GHC's primary care clinics are National Committee for Quality Assurance (NCQA) level III patient-centered medical homes, and NCQA performance measures are tracked electronically and used to improve preventive screening and chronic disease management through targeted outreach., ${ }^{2,3}$ Clinical work is shared such that team members work to their highest level of training. Virtual medicine is emphasized and over $70 \%$ of all physician-patient encounters occur via telephone or secure message. ${ }^{3}$ This advanced model of care delivery creates an ideal learning environment, allowing residents to experience first hand the essential elements of highfunctioning primary care.

At my first visit with Beth-Ann, we created a care plan and organized a team to address her needs. I shared the plan with our nurse who assists in the management of hypertension and diabetes, virtually consulted with specialists for the patient's palpitations and rectal bleeding, and relied on my medical assistant to attend to her preventive care. After receiving prompt responses from all team members, I spoke with BethAnn to co-create a time line to address her many health concerns.

Despite the primary care delivery innovations at GHC, our old curriculum was all too familiar. Learning experiences were divided into four-week block rotations in which residents were completely absent from clinic during intensive inpatient blocks. These gaps, combined with only $20 \%$ of total training time spent in clinic, contributed to poor patient access and low resident and patient satisfaction in clinic. As extreme part-time providers, residents did not function as full team members, had reduced confidence in their outpatient skills, and carried high stress from frequently seeing high-needs patients for singular visits.

These problems led to conversations among faculty and residents on strategies to transform clinic into an environment in which residents would feel their greatest sense of belonging and mastery. We believed that the quality of the 
outpatient experience would improve for both residents and patients if training more authentically mimicked the work-life of a primary care physician. Thus, our new curriculum's mission became to have each resident care continuously for the primary care needs of a panel of 500 patients, and to hold our resident clinic to GHC-wide quality standards.

To achieve this, we needed to address the limitations in our previous training model. First, curricular time spent in sub- specialty training where residents were non-essential staff was reduced in order to increase resident continuity clinic to $30 \%$ of our total residency training (Fig. 1a). Residents now graduate having experienced over 350 half-day sessions in their continuity clinic, $30 \%$ greater than previously. Next, resident clinic schedules were revised to prevent long absences, and block rotations were eliminated in favor of a longitudinal schedule. For example, adult medicine inpatient training now occurs in one-week bursts every six weeks (see Fig. 2 for an
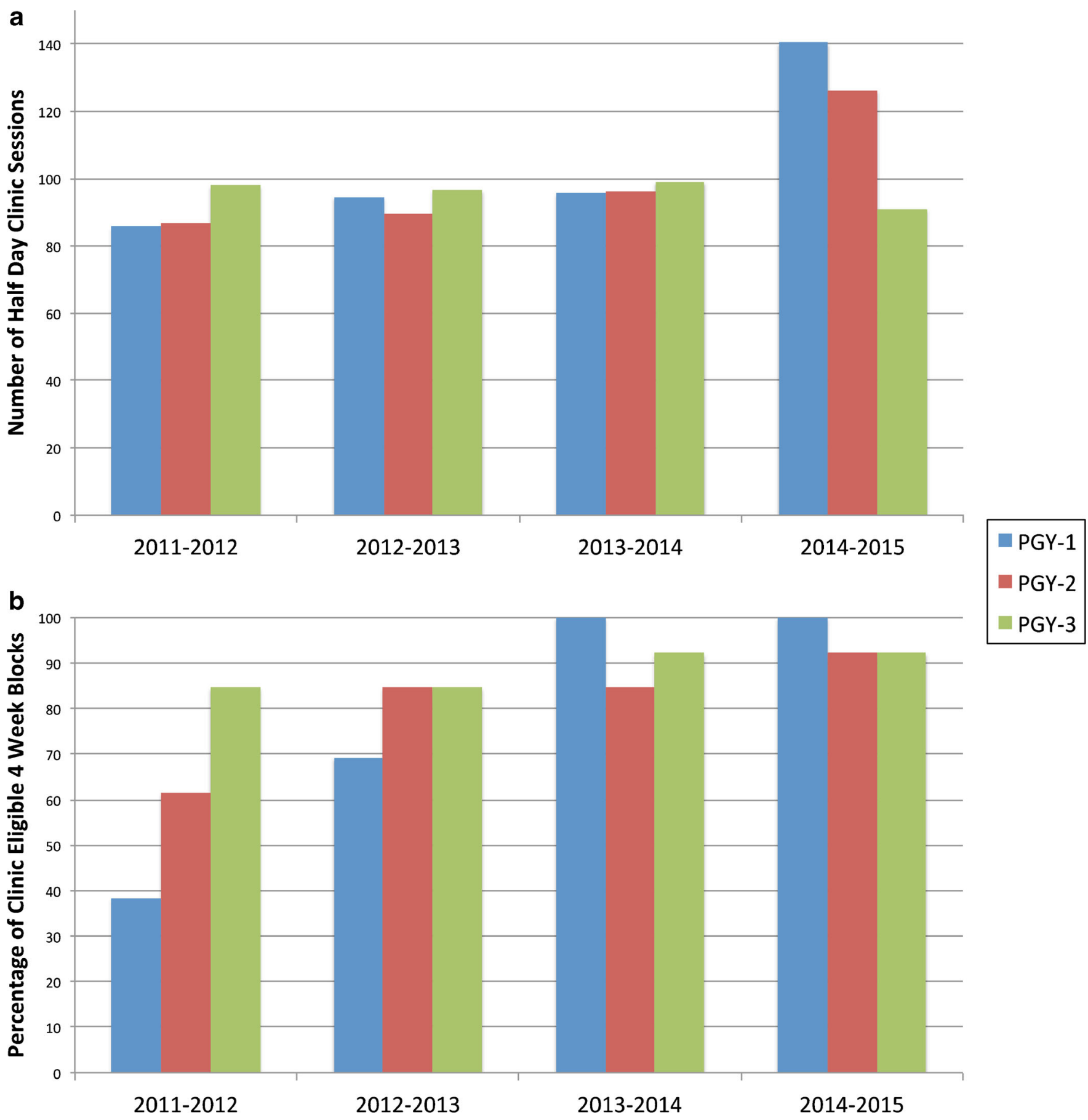

Figure 1 (a) Total half-day clinic sessions by post-graduate year measured before, during, and after curriculum revisions. (b) Percentage of four-week periods in which residents have scheduled clinic sessions. *Abbreviations: PGY=Post-Graduate Year. 
Sun.
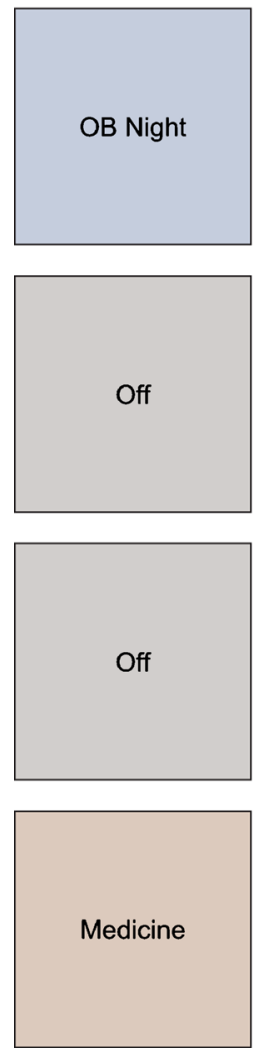

Mon.
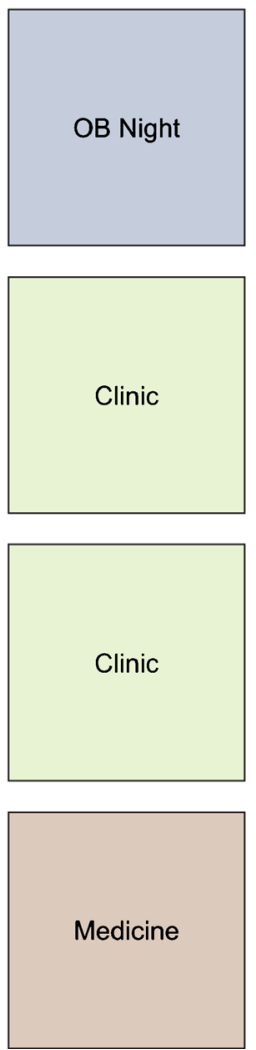

Tues.
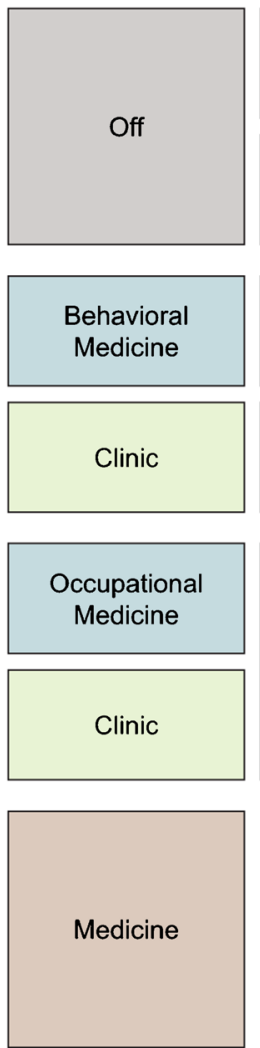

Fri.
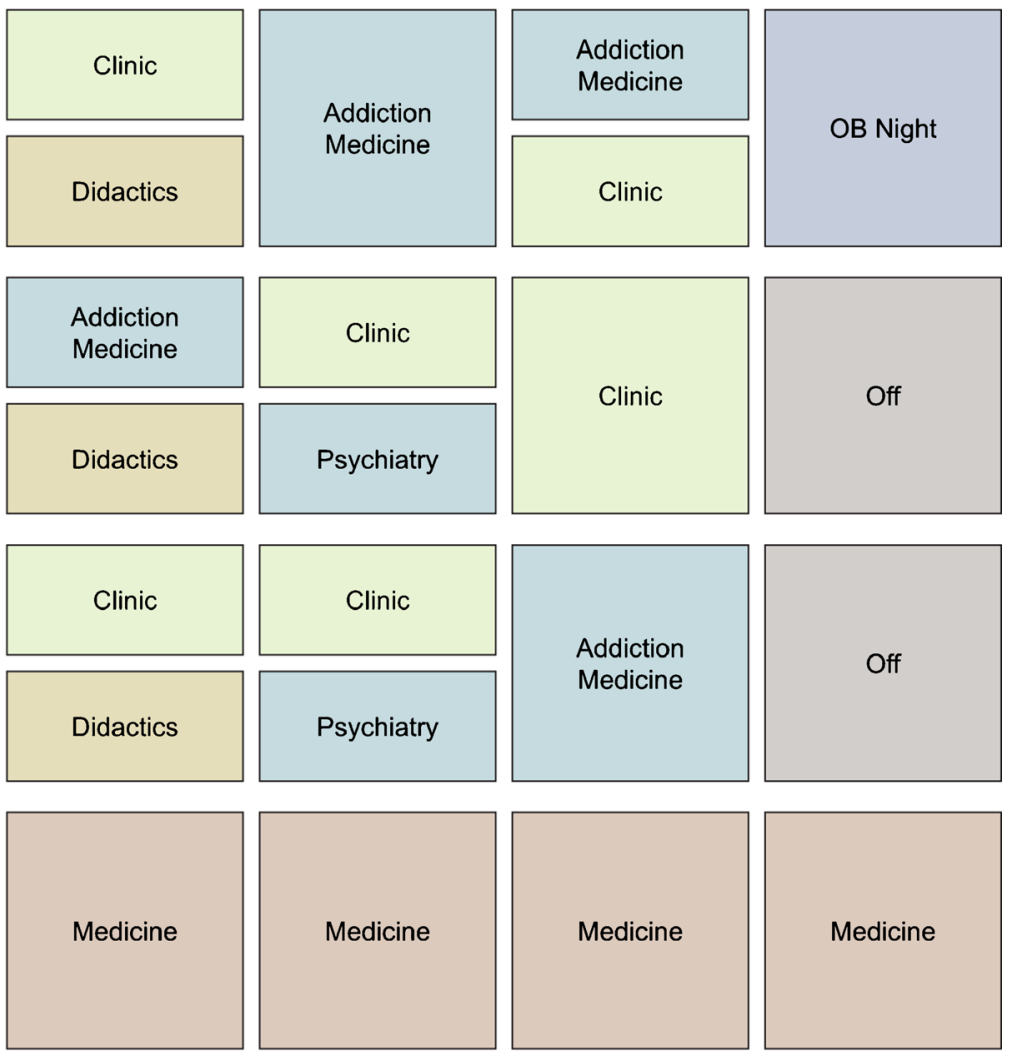

Sat.
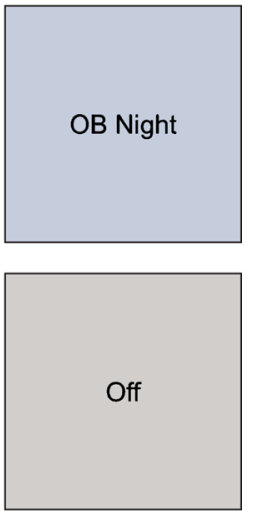

Figure 2 Example intern schedule in the new curriculum. *Abbreviations: $O B=O b s t e t r i c s$. example schedule). There are now only two four-week blocks throughout all of residency when residents are not regularly scheduled in their continuity clinic (Fig. 1b), and only one four-week rotation in which residents combine inpatient duties with afternoon clinics.

Additionally, we enhanced the formal education of outpatient skills. Residents now receive training in panel management and data review, and have regular time with their medical assistant to fill care gaps in their patient panels through outreach. A new virtual medicine curriculum teaches skills such as writing secure messages, conducting phone visits, and managing the electronic inbox. Residents manage their electronic inbox daily or rely on a resident coverage schedule. Finally, with the many changes, feedback is emphasized. Residents receive feedback during and after each clinic session, as well as bi-annually from all team members.

Over the following months, my relationship with BethAnn deepened through a mixture of in-person and virtual visits. I began to fit her complex medical issues into a larger story that spoke to the social and financial challenges she faced. My initial feeling of being overwhelmed was replaced by a sense of confidence in my ability to care for her. When she confided in a secure message that she was having new pain symptoms that kept her from eating, I appreciated the deviation from her baseline chronic pain and advised her to schedule an in-person appointment. At her visit later that week, I diagnosed trigeminal neuralgia and began treatment. Later, when her Hemoglobin Alc showed a three-point drop, we celebrated. "We did this together," she said. I felt joy in our shared victory.

The full complement of changes to the curriculum was implemented over a three-year period and took full effect in July 2014. While the long-term impact remains undetermined, interns report having continuity and meaningful relationships with their panel of patients in just their third month of residency and feel that clinic is their learning home. Transitioning inpatient duties from four-week to one-week blocks has 
received positive reviews from residents, though the effect on learning needs ongoing evaluation.

In actualizing our new curriculum, scheduling continues to be our greatest challenge. After the large initial time and energy investment in redesign, we now face the question of how to set the pace of work in our threeyear longitudinal curriculum. Residents now transition into full-spectrum primary care on their entrance into residency rather than on graduation, and the mastery of core skills and competencies has a new cadence. As such, trainee expectations are continually being reviewed and revamped. Additionally, logistical issues continue to arise when arranging vacations, scheduling sub-specialty training, and covering when residents are unexpectedly absent.

Though our program's experience is unique, we believe it is applicable to other primary care residencies. First, our increased clinic curricular time was created without reducing the absolute amount of inpatient training time. While the requirements of a family medicine resident ultimately differ from those of an internal medicine or pediatric resident, core principles of outpatient training gained by our model remain equally important. Further, while our residency clinic has the capacity to provide high-level outpatient care using tools that are not yet widely available, these services are increasingly being incentivized by national health policy changes. ${ }^{4}$ This heralds an opportunity to create advanced primary care practices structured to incorporate both patient care and resident educational needs. For instance, resident schedules can be crafted to promote patient access and provider continuity, and staff schedules can simultaneously be matched to residents to foster stronger care team relationships.
In just 14 months of residency, I have had over 30 discrete virtual and in-person clinical encounters with Beth-Ann. Her multiple medical issues have unfolded over time, challenging me to develop new clinical and organizational skills practiced over multiple mediums. Through providing care for my entire panel of patients, I hone complex skills distinct from those that I learn in the inpatient setting. Importantly, it is my relationship with these patients that brings joy to my work and motivates me to pursue a career in primary care. By providing trainees with a positive ambulatory experience, the GHC model helps meet the national need for welltrained primary care physicians, and may ultimately attract more students to careers in primary care.

Acknowledgements: Contributors: Paul Ford, MA, Residency Analyst, Group Health Cooperative

Conflicts of Interest: The authors declare that they do not have any conflicts of interest.

Corresponding Author: Kathleen Barnes, MD, MPH; Group Health Cooperative, 125 16th Avenue East, Suite CSB 4, Seattle, WA 98112 , USA (e-mail: Barnes.Kathleen@ghc.org).

\section{REFERENCES}

1. Holmboe EES, et al. Reforming Internal Medicine Training. J Gen Intern Med. 2005;20(12):1165-72.

2. Larson, EB. Group Health Cooperative - One Coverage and Delivery Model for Accountable Care. NEJM 361;17, October 22, 2009: 1620-22.

3. Reid R, et al. Spreading a Medical Home Redesign: Effects on Emergency Department Use and Hospital Admissions. Annals of Family Medicine. 2013;11:S19-26.

4. Patient Protection and Affordable Care Act, 42 U.S.C. 18001 et seq. 2010. 